\title{
Precipitation of Bioactive Ingredients from Extracted Centella asiatica via Gas Anti-Solvent Technique
}

\author{
Manop Charoenchaitrakool ${ }^{1,2}$, Ornkanit Chantes ${ }^{1}$ \\ ${ }^{1}$ Department of Chemical Engineering, Faculty of Engineering, Kasetsart University \\ Bangkok 10900, Thailand \\ ${ }^{2}$ Center for Advanced Studies in Nanotechnology for Chemical, Food and Agricultural Industries \\ KU Institute for Advanced Studies, Kasetsart University \\ Bangkok 10900, Thailand \\ manop.c@ku.ac.th
}

\section{Extended Abstract}

Centella asiatica L. (CA) is one of the important medicinal plants widely used as folk medicine to treat a wide range of illnesses, such as headaches, ulcers, asthma, eczemas, tumors and cancer, diabetes and memory loss [1]. The primary active ingredients of CA consist of flavonoid glycosides, free amino acid, polyacetylenic compounds and triterpene saponins [2]. Main compounds in triterpene saponins, namely madecassoside, asiaticoside, madecassic acid and asiatic acid are considered as bioactive compounds. These bioactive compounds are well known for their antioxidant, antibacterial and fungicidal activity against fungi $[3,4]$. In general, the use of Centella asiatica as herbal remedy requires large amount of unprocessed materials due to low concentrations of bioactive compounds present in CA leaves. Therefore, it would be beneficial to extract the bioactive compounds and use the extracts as a concentrated medicine.

The aim of this research was to precipitate bioactive ingredients from Centella asiatica using Gas Anti-Solvent (GAS) process. In this research, active ingredients were extracted from Centella asiatica dried powder by maceration method at room temperature. The extracted solution was then used as a starting solution for the GAS precipitation process with the use of carbon dioxide as an anti-solvent. The effects of \% ethanol in the mixed solvent of ethanol and methanol (0-100\%), carbon dioxide flow rate $(3-9 \mathrm{~mL} / \mathrm{min})$ and temperature $\left(25-45^{\circ} \mathrm{C}\right)$ on the amount of precipitates were investigated. The Box-Behnken design of experiments was carried out using the MINITAB 17. The precipitated product was analyzed using HPLC and tested for antioxidant activity (via DPPH and ABTS assays). The amount of total phenolic was also determined. The results show that \% ethanol in the mixed solvent of ethanol and methanol in the maceration step ( $\mathrm{S}$ and $\mathrm{S} * \mathrm{~S})$ were significant parameters for madecassoside, asiaticoside and total triterpene saponins $(\mathrm{p}<0.05)$. Moreover, for the case of total triterpene saponins, the precipitation temperature $(\mathrm{T})$ was also found to be significant variable. For madecassic acid and asiatic acid, it was found that all coefficient terms were not significant $(p>0.05)$. Higher amounts of active ingredients could be precipitated when a lower percentage of ethanol in the mixed solvent was used in the maceration step. Moreover, an increase in precipitation temperature resulted in a reduction in the amount of precipitates. At the optimal conditions, the amount of madecassoside, asiaticoside, madecassic acid and asiatic acid were found to be $5,733 \pm 537,2,948 \pm 249,1,678 \pm 163$ and $436 \pm 17 \mu \mathrm{g} / 5 \mathrm{~mL}$ of extracted solution, respectively. In the antioxidant studies, the highest Trolox equivalent found in the products using DPPH and ABTS assays were 5,622 \pm 336 and $10,729 \pm 820 \mu \mathrm{g} / 5 \mathrm{~mL}$ of extracted solution. The highest total phenolic was found to be $3,653 \pm 49 \mu \mathrm{g} / 5 \mathrm{~mL}$ of extracted solution.

\section{References}

[1] C. Niamnuy, M. Charoenchaitrakool, P. Mayachiew and S. Devahastin, "Bioactive compounds and bioactivities of Centella asiatica (L.) urban prepared by different drying methods and conditions," Drying Technol., vol. 31, pp. 20072015, 2013.

[2] R. Srivastava, Y. N. Shukla and S. Kumar, "Chemistry and pharmacology of Centella asiatica: A review," J. Med. Arom. Plant Sci., vol. 19, pp. 1049-1056, 1997.

[3] V. Prakash, N. Jaiswal, and M. Srivastava, "A review on medical properties of Centella Asiatica," Asian J. Pharm. Clin. Res., vol. 10, pp. 69-74, 2017.

[4] M. Restuati and D. S. Diningrat, "Antimicrobial profile of premna pubescens. Blume and centella asiatica extracts against bacteria and fungi pathogens," Int. J. Pharmacology, vol. 14, pp 271-275, 2018. 